80 percent of normal, distorted taste, hallucinated metallic taste, and BMS. Upon application of an ice cube to the tongue, both the metallic taste and the BMS resolved for a few seconds, without impairing her true taste ability. With repeat application, the alleviation effect persists.

RESULTS: Abnormalities in Neurologic Examination: Sensory Examination: Decreased pinprick and temperature bilateral lower extremities. Reflexes: $3+$ throughout. Bilateral positive Hoffman's reflexes. Chemosensory testing: Olfaction: Brief Smell Identification Test: 9 (normosmia). Retronasal Smell Index: 10 (normosmia). Gustation: Propylthiouracil Disc Taste Test: 5 (normogeusia).

DISCUSSION: Transient Receptor Potential 5, is expressed in tongue taste buds, facilitating sweet perception, and is temperature dependent (Fujiyama, 2010). Ice may act to reduce such sweet taste receptor discharge, causing an imbalance in taste fiber discharge thus inhibiting the perceived metallic taste. In those who suffer from intractable phantogeusia, a trial of ice cubes or mechanisms to reduce temperature of the tongue is warranted.

83

\section{Catatonia in a 17-year-old Male Patient with Bipolar Disorder, a Case Study}

Sultana Jahan, $M D$

Associate Professor, Department of Psychiatry, University of Missouri-Columbia, Columbia, Missouri

ABSTRACT: Study Objective(s): Catatonia is not only present in adults; children \& adolescents can suffer from catatonia but are often misdiagnosed. A study by Ghaziuddin, Dhossche and Marcotte (2012) found that 18 of the 101 child and adolescent patients had symptoms of catatonia, but only 2 actually had been given a diagnosis by their providers.

METHOD: 17-year-old male who was recently discharged from the inpatient psychiatric unit with the diagnosis of Major Depressive Disorder. His discharge medication was bupropion XL $150 \mathrm{mg}$ daily. Within 10 days of his discharge, he was back to the emergency room with worsening anxiety and manic symptoms. At the emergency room, patient's sister reported that he was acting differently within the last 3-4 days, and making statements that he can save the world that everyone was talking about him. He was also speaking faster than usual, having decreased need for sleep. He reported hearing voices, seeing things. Patient was admitted again, and was given diagnosis of Bipolar Mood Disorder, type I, manic phase, with psychosis. He was started on divalproex $500 \mathrm{mg}$ bid for mood stabilization. His Bupropion was discontinued Gradually his Divalproex was increased to $750 \mathrm{mg}$ bid. During his hospital stay he developed lack of spontaneous speech, sluggish responses to questions with automatic answers such as "I don't know". He also developed very sluggish motor movements. There was negativism. He needed one on one support for his daily activities of living, needed step by step instructions for all ADLs.

All the test results were negative including EEG, MRI and CT scan of the brain. Bush Francis catatonia rating scale was done and he scored 15. Lorazepam Challenge Test was performed, and the scale was repeated after the patient was given an IM dose of $2 \mathrm{mg}$ of Lorazepam, and he scored 2. At this point catatonia diagnosis was confirmed. He was started on scheduled doses of Lorazepam, gradually his Lorazepam dose was increased up to $9 \mathrm{mg}$ per day. His catatonia responded to Lorazepam treatment.

RESULTS: 17 year old male who initially hospitalized for symptoms of MDD and discharged with antidepressant, came back to the ER within 10 day with symptoms of mania with psychosis. During in-patient's 2nd in-patient stay he developed catatonia, which was promptly diagnosed and appropriately treated with Lorazepam.

CONCLUSIONS: Catatonia can happen in children \& adolescents with mood disorders, or with other psychiatric or medical conditions. Timely diagnosis and treatment is very crucial to avoid poor outcome, especially because treatment options for catatonia are well understood; Benzodiazepines, electroconvulsive therapy and reduction or discontinuation of antipsychotics are successful in the treatment of catatonia (Ghaziuddin, Dhossche and Marcotte, 2012).

\section{REFERENCE:}

Fink M. (2009). Catatonia: A Syndrome Appears, Disappears, and Is Rediscovered. The Canadian Journal of Psychiatry, 54(7), 437-445.

\section{4}

\section{Assessment of Current Clinical Practices in Recognizing and Treating Bipolar Disorder}

Christoph U. Correll, $M D^{\prime}$; Piyali Chatterjee ${ }^{2}$; Susan H. Gitzinger, PharmD, MPA ${ }^{2}$; Jovana Lubarda, $P h D^{2}$; and Marcello Morgan, $\mathrm{MD}, \mathrm{MPH}^{2}$

${ }^{1}$ Professor of Psychiatry and Molecular Medicine; Hofstra Northwell School of Medicine; Hempstead, New York; Investigator, Center for Psychiatric Neuroscience; Feinstein Institute for Medical Research; Manhasset, New York; Medical Director, Recognition and Prevention (RAP) Program; The Zucker Hillside Hospital, Department of Psychiatry; Glen Oaks, New York

${ }^{2}$ Medscape Education, New York, NY 
ABSTRACT: Background: Among adults in the US, bipolar disorder affects $2.6 \%$ or 5.7 million individuals; $83 \%$ of cases are considered to be severe. Even when an accurate diagnosis of bipolar disorder is established, its treatment remains suboptimal, and those with the disorder often fail to receive any care or evidence-based care.

INTRODUCTION: A continuing medical education (CME)certified 25-item, multiple choice clinical practice assessment survey was developed to assess recognition and treatment of bipolar disorder, specifically, the use of LAIs in these patients.

METHODS: The survey included knowledge- and casebased multiple-choice questions completed confidentially online. The survey was launched on December 20, 2017 and hosted on the Medscape Education website. Participant responses were collected through January 31, 2018. Confidentiality was maintained, and responses were de-identified and aggregated before analyses.

RESULTS: ( $\mathrm{n}=1123$ psychiatrists; 305 primary care physicians [PCPs]):

- When asked about assessment tools in bipolar disorder, only $43 \%$ of psychiatrists and $36 \%$ of PCPs could identify the correct use of the MDQ screening instrument, while only $64 \%$ of psychiatrists and $51 \%$ of PCPs knew that the use of the MDQ can improve recognition of bipolar disorder in patients with depression;

- Psychiatrists were more likely to correctly identify the symptoms that most strongly support a diagnosis of bipolar disorder compared to PCPs $(76 \%$ vs $43 \%$, respectively);

- When asked about laboratory testing in mood disorders, $52 \%$ of psychiatrists and $46 \%$ of PCPs knew that laboratory testing can help exclude alternative causes for mood symptoms;

- The majority of both healthcare professionals $(73 \%$ $75 \%$ ) did not know that diagnosis of bipolar I disorder relies heavily on changes in activity, energy, and mood; - $87 \%$ of psychiatrists and $76 \%$ of PCPs did not identify oral aripiprazole as the only SGA not approved by the FDA for the maintenance treatment of bipolar I disorder;

- $49 \%$ of PCPs did not recognize lithium as the first choice for maintenance monotherapy for bipolar I disorder according to the guidelines;

- Only $19 \%$ of psychiatrists and $20 \%$ of PCPs correctly chose aripiprazole monohydrate and risperidone microspheres as the LAI SGAs indicated for use as monotherapy for patients with bipolar I disorder;

- When asked what is the most common barrier to prescribing LAI antipsychotics in patients with bipolar disorder, 34\% of psychiatrists selected "Patients fear of injectables"

CONCLUSIONS: This educational research identified psychiatrists and PCPs' current real-world clinical practices and gaps in the knowledge and competence in the diagnosis and assessment of bipolar disorder, and the treatment options for this condition. Further educational efforts tailored to address identified gaps for each audience are warranted.

Funding Acknowledgements: Otsuka

86

\section{Hyperfamiliarity for Unknown Faces in a Patient with Lewy Body Dementia}

Aghaegbulam Uga, $M D^{\prime}$; and Terngu Ibilah, $M D^{2}$

${ }^{1}$ Associate Professor and Program Director, Psychiatry and Internal Medicine Combined Program, Texas Tech University Health Science Center, El Paso, TX

${ }^{2}$ PGY 2 Resident, Psychiatry and Internal Medicine Combined Program, Texas Tech University Health Science Center, El Paso, TX

ABSTRACT: Title: "I know everybody here"

Hyperfamiliarity for unknown faces, a delusion of misidentification, is a rare disorder.

We present a 67-year-old female admitted with worsening cognitive impairment and poor self-care associated with parkinsonian symptoms of one-year duration. During evaluation, she was noted to relate to strangers with familiar gestures like people she already knew causing distress for family and care givers.

Workup revealed significant cognitive impairment, MOCA of $9 / 30$ and neuroimaging showing diffuse temporal lobe volume loss predominantly on the left.

Assessment was Lewy body dementia with hyperfamiliarity for unknown faces.

This rare presentation reflects the need for detailed examination and workup during evaluation.

87

\section{Efficacy Measures in an Open-label Dose- Optimization of an Amphetamine Extended- Release Oral Suspension in Children with Attention-Deficit/Hyperactivity Disorder}

Andrew Cutler, $\mathrm{MD}^{\prime}$; Antonio Pardo, $M D^{2}$; Thomas R. King, $M S, \mathrm{MPH}^{2}$; Judith C. Kando, PharmD, BCPP and and Barry K. Herman, $M D, M M M^{2}$

${ }^{1}$ Meridien Research, Bradenton, FL

2 Tris Pharma, Inc., Monmouth Junction, NJ 